\title{
Apoptosis inhibition is involved in improvement of sevoflurane-induced cognitive impairment following normobaric hyperoxia preconditioning in aged rats
}

\author{
YING WANG $^{1,2}$, CHUN-PING YIN ${ }^{1}$, YAN-LEI TAI ${ }^{1}$, ZI-JUN ZHAO ${ }^{3}$, ZHI-YONG HOU $^{4}$ and QIU-JUN WANG ${ }^{1}$ \\ ${ }^{1}$ Department of Anesthesiology, The Third Hospital of Hebei Medical University, Shijiazhuang, Hebei 050051; \\ ${ }^{2}$ Department of Anesthesiology, Tangshan Gongren Hospital, Tangshan, Hebei 063000; \\ ${ }^{3}$ Department of Anesthesiology, Hebei Chest Hospital; ${ }^{4}$ Department of Orthopaedic Surgery, \\ The Third Hospital of Hebei Medical University, Shijiazhuang, Hebei 050051, P.R. China
}

Received June 19, 2020; Accepted December 4, 2020

DOI: 10.3892/etm.2021.9636

\begin{abstract}
Sevoflurane, a commonly used anesthetic agent has been confirmed to induce cognitive impairment in aged rats. Normobaric hyperoxia preconditioning has been demonstrated to induce neuroprotection in rats. The present study aimed to determine whether normobaric hyperoxia preconditioning could ameliorate cognitive deficit induced by sevoflurane and the possible mechanism by which it may exert its effect. A total of 66, 20-month-old male Sprague-Dawley rats were randomly divided into 3 groups ( $\mathrm{n}=22$ each): Rats in the control (C) and sevoflurane anesthesia (S) groups received no normobaric hyperoxia preconditioning before sevoflurane exposure, rats in the normobaric hyperoxia pretreatment $(\mathrm{HO})$ group received normobaric hyperoxia preconditioning before sevoflurane exposure (95\% oxygen for 4 continuous h daily for 6 consecutive days). The anesthesia rats ( $\mathrm{S}$ and $\mathrm{HO}$ groups), were exposed to $2.5 \%$ sevoflurane for $5 \mathrm{~h}$, while the sham anesthesia rats (C group) were exposed to no sevoflurane. The neurobehavioral assessment was performed using a Morris water maze test, the expressions of the apoptosis proteins were determined using western blot analysis, and the apoptosis rate and cytosolic calcium concentration were measured by flow cytometry. Normobaric hyperoxia preconditioning improved prolonged escape latency and raised the number of platform
\end{abstract}

Correspondence to: Dr Qiu-Jun Wang, Department of Anesthesiology, The Third Hospital of Hebei Medical University, 139 Ziqiang Road, Shijiazhuang, Hebei 050051, P.R. China

E-mail: wangqiujunsy@163.com

Abbreviations: ANOVA, one-way analysis of variance; $\left[\mathrm{Ca}^{2+}\right]_{c}$, cytosolic calcium concentration; DMEM, Dulbecco's modification Eagle's medium; LORR, loss of the righting reflex; MWM, Morris water maze; NBO, normobaric hyperoxia; NBO-PC, normobaric hyperoxia preconditioning; PI, propidium iodide; POCD, postoperative cognitive dysfunction; PS, phosphatidylserine

Key words: normobaric hyperoxia, apoptosis, aged, sevoflurane, POCD crossings induced by sevoflurane in the Morris water maze test, increased the level of bcl-2 protein, and decreased the level of bax and active caspase- 3 protein, the apoptosis rate and cytosolic calcium concentration in the hippocampus $24 \mathrm{~h}$ after sevoflurane exposure. The findings of the present study may imply that normobaric hyperoxia preconditioning attenuates sevoflurane-induced spatial learning and memory impairment, and this effect may be partly related to apoptosis inhibition in the hippocampus. In conclusion, normobaric hyperoxia preconditioning may be a promising strategy against sevoflurane-induced cognitive impairment by inhibiting the hippocampal neuron apoptosis.

\section{Introduction}

As the global population ages, and with improvements in medical and health technologies, there are more elderly patients and more are requiring surgical interventions and procedures (1). Globally, 50\% of all elderly individuals are estimated to undergo at least one surgical procedure (2). Postoperative cognitive dysfunction (POCD) is a complication of anesthesia and surgery associated with significant morbidity and even mortality that is widely considered an important clinical problem, particularly in elderly patients. In a prospective multicenter trial performed by ISPOCD (international study of postoperative cognitive dysfunction) in 1998, the incidence of POCD in patients undergoing non-cardiac surgery was present in $26 \%$ at 1 week, and approximately $10 \%$ of the patients still had cognitive dysfunction after 3 months (3). Currently, there is no effective clinical treatment for POCD (4) and an urgent need to develop a novel treatment strategy to improve the prognosis of this condition (5).

The risk and precipitating factors of POCD are multifarious including increasing age, low education level, burden of illness, pain, anesthesia, repeated surgeries, postoperative infections and respiratory complications (6). Especially volatile anesthetics, one class of the most widely used drugs since the 19th century for general anesthesia is often mentioned as a possible cause of POCD (7). For instance, isoflurane (8), sevoflurane (9) and desflurane (10), which are commonly used 
in clinic, have been found to cause cognitive impairment. Previous studies have provided evidence of neurotoxicity caused by volatile anesthetics (11-13), and the safety of these anesthetic agents has come under scrutiny (14). Impairment of memory and spatial learning were observed in aged rodents receiving volatile anesthetics including sevoflurane (12). Although the mechanisms underlying the volatile anesthetic neurotoxicity have not been clarified, neuroapoptosis (15), neuroinflammation (16) and neurodegeneration (17) may be involved.

Normobaric hyperoxia (NBO) preconditioning (PC) has been demonstrated to protect against heart and cerebral ischemia as well as renal ischemia (18-20). Similarly, NBO-PC also attenuates cognitive impairment in an Alzheimer's disease mouse model (21). The protective effects of NBO-PC are attributed to antiapoptotic and anti-inflammatory effects (22). Since apoptosis and inflammation are involved in the neurotoxicity induced by volatile anesthetics $(15,16)$, NBO-PC may be an effective and feasible method to alleviate cognitive impairment related to volatile anesthetics including sevoflurane.

Thus far, to the best of our knowledge whether NBO-PC can ameliorate cognitive deficit induced by sevoflurane and the possible mechanism by which it may exert its effect has not yet been clarified. We previously found that sevoflurane induces apoptosis in hippocampal neurons and causes cognitive deficit in aged rats (15). Based on these findings, it was hypothesized that NBO-PC may ameliorate cognitive deficit induced by sevoflurane through inhibiting hippocampal apoptosis. In the present study, this hypothesis was tested in an aged rat model of cognitive dysfunction induced by sevoflurane and the underlying molecular mechanism of this phenomenon was explored. In brief, if the neuroprection of NBO-PC could be proved, it may indicate a potential novel target for the treatment of POCD.

\section{Materials and methods}

Study design. The present study was a prospective, randomized, controlled animal study, which started in September 2018 and ended in October 2019, with the duration of the animal experimentation for 5 months. It was approved by the Ethics Committee for Animal Experimentation (Ethical approval no. Guo A2017-026-1), and the animals were studied at Hebei Medical University (Shijiazhuang, China).

Animals. A total of 66 male Sprague-Dawley 20-month-old rats (450-550 g) were divided randomly into 3 groups ( $\mathrm{n}=22$ each): i) Control group (C group); ii) sevoflurane group ( $\mathrm{S}$ group); and iii) sevoflurane + NBO-PC group (HO group). To adapt to the animal care facility for 5 days before the experiment, these rats were housed in plastic cages under standard conditions, with free access to food and water, and maintained on a $12 / 12 \mathrm{~h}$ light/dark cycle. The rational for choosing rats of this age was previous evidence that sevoflurane inhalation resulted in learning and memory deficits (23). There was no death in each group prior to decapitation.

Preconditioning protocol and experimental procedure. NBO-PC was performed as previously described (24). Briefly, animals assigned to the HO group received $4 \mathrm{~h}$ of $\mathrm{NBO}$ treatment at 1 atm absolute (ATA) in $95 \%$ oxygen each day for 6 consecutive days in environmental chambers comprised air-tight boxes $(65 \times 25 \times 45 \mathrm{~cm})$ with gas inlet and outlet ports at room temperature $\left(22 \pm 1^{\circ} \mathrm{C}\right)$. The $\mathrm{C}$ and $\mathrm{S}$ groups were exposed to normobaric normoxia ( $21 \%$ oxygen) according to the same protocol. After the last preconditioning (the 6th preconditioning cycle) $24 \mathrm{~h}$ later, rats assigned to the $\mathrm{S}$ and HO groups were placed in sealed transparent anesthesia induction chambers with soda lime at the bottom near the side opening of where the anesthetic machines were connected. The aforementioned rats received 2.5\% sevoflurane (Maruishi Pharmaceutical Co., Ltd.) via humidified $50 \% \mathrm{O}_{2}$ carrier gas from a calibrated vaporizer for $5 \mathrm{~h}$. Rats in the $\mathrm{C}$ group were also placed in the same chambers for $5 \mathrm{~h}$ with no sevoflurane given. Following $24 \mathrm{~h}$ sevoflurane-exposure, rats were subdivided for the Morris water maze (MWM) test, apoptosis detection in the hippocampus and cytosolic calcium concentration measurement of hippocampal cells. The experimental procedure was shown in Fig. 1.

Morris water maze test. Cognitive function of 10 rats randomly selected from each group was tested using the MWM test $24 \mathrm{~h}$ after sevoflurane exposure. The MWM (Shanghai Jiliang Software Technology Co., Ltd.) was a circular pool $(180 \mathrm{~cm}$ diameter, $50 \mathrm{~cm}$ depth) with black walls and a water $\left(23 \pm 1^{\circ} \mathrm{C}\right)$ depth of $30 \mathrm{~cm}$, which was divided into 4 equal quadrants. A hidden round platform (10 $\mathrm{cm}$ diameter) was submerged $2 \mathrm{~cm}$ below the surface of water, located in one quadrant. The MWM test consisted of 5 days of place navigation test (a test of ability to learn the location of the hidden platform) and a probe trial (a test of ability to remember the previously-learned location of the escape platform having been removed) on the 6th day. In the test, rats were given 4 trials/day for 5 consecutive days. A trial was terminated and recording was stopped when the animal reached the platform, where it was allowed to remain for $15 \mathrm{sec}$. If the rat failed to find the target before $90 \mathrm{sec}$, it was usually guided to the goal and allowed to stay on the platform for $15 \mathrm{sec}$ with the escape latency being recorded as $90 \mathrm{sec}$. On the sixth day, the hidden platform was removed and the rat was placed in the opposite quadrant. Each rat was allowed to swim freely in the pool for $90 \mathrm{sec}$ and the platform crossing times were recorded.

Measurements of apoptosis rate and cytosolic calcium concentration $\left(\left[\mathrm{Ca}^{2+}\right]_{c}\right)$ in the hippocampus. Apoptosis rate of the hippocampus was measured by flow cytometry with an Annexin-V-FITC/propidium iodide (PI) apoptosis detection kit (Vazyme Biotech Co. Ltd.). Phosphatidylserine (PS) normally located on the inner side of the cell membrane, flips to the outer side of the cell membrane at the early stage of apoptosis (24). Annexin-V, a $\mathrm{Ca}^{2+}$ dependent phospholipid-binding protein, with affinity for PS is routinely used to label externalization of PS (25). PI (propidium iodide, a nucleic acid dye), which does not permeate cells with an intact plasma membrane, can penetrate the cell membrane to make the nucleus red in the late stage of apoptosis and necrosis (26). Annexin-V and PI dual staining allows discrimination of apoptotic cells (Annexin- $\mathrm{V}^{+}, \mathrm{PI}^{-}$), late apoptotic/necrotic cells (both Annexin- $\mathrm{V}^{-}$and $\mathrm{PI}^{+}$) and live cells (both Annexin- $\mathrm{V}^{-}$and $\mathrm{PI}^{-}$) (27). A total of 6 rats randomly selected from each group, $24 \mathrm{~h}$ after sevoflurane-exposure 


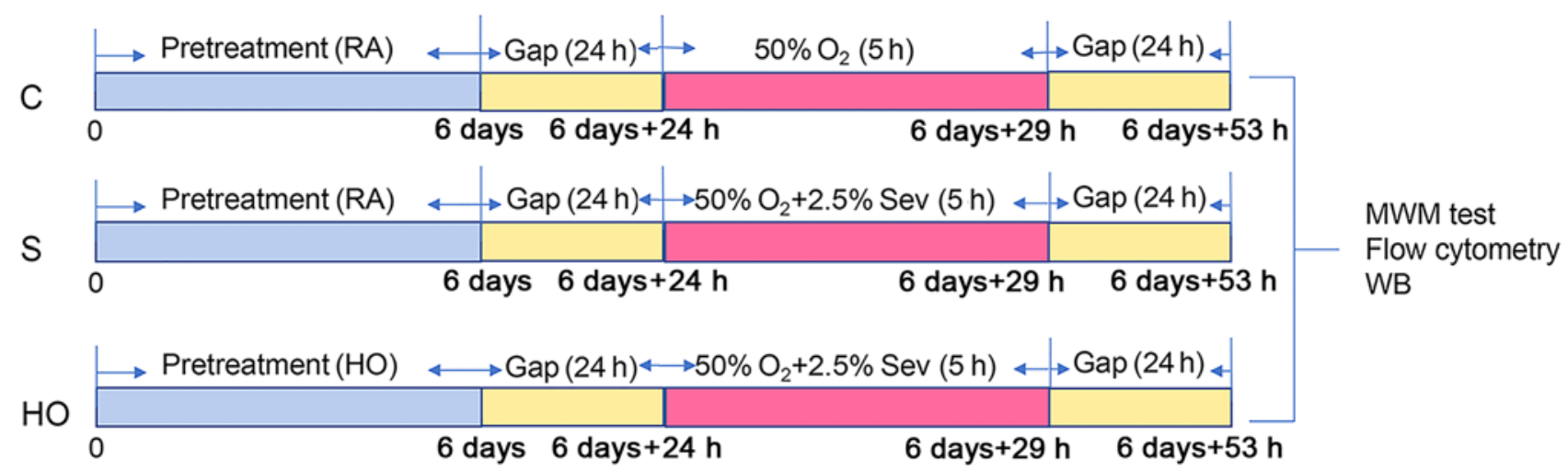

Figure 1. Experimental protocol for the study of normobaric hyperoxia preconditioning. RA, normoxia; HO, normobaric hyperoxia; Gap, ordinary air room without any procedure; d, day; h, hour; Sev, sevoflurane; WB, western blotting; MWM, Morris water maze; C group, control group; S group, sevoflurane group; HO group, sevoflurane + NBO-PC group; NBO-PC, normobaric hyperoxia preconditioning.

were anesthetized with an intraperitoneal injection of $10 \%$ chloral hydrate $(250 \mathrm{mg} / \mathrm{kg})$ without any signs of peritonitis observed. According to previous literature (28), the loss of the righting reflex (LORR, an indicator of hypnosis), ambulation and voluntary movement were considered to be indicators of the success of chloral hydrate anesthesia. LORR was assessed by attempting to place the rat in left lateral recumbency, followed by dorsal recumbency. If the rat remained on its back for $10 \mathrm{sec}$, LORR was considered to be achieved (29). After successful induction of chloral hydrate, A total of 18 rats from three groups were sacrificed by decapitation. Brain tissue was immediately removed and the left hippocampi were separated and cut into blocks. Hippocampal tissue $(\sim 100 \mathrm{mg})$ was placed on a 100-mesh copper net and cut up with tweezers and then gently rubbed and filtered. The filtrate was centrifuged at $167.7 \mathrm{x}$ g for $5 \mathrm{~min}$ at room temperature. Cells collected after centrifugation were resuspended in $500 \mu \mathrm{l}$ Annexin-V binding buffer to prepare a single cell suspension $\left(1-5 \times 10^{5} / 1\right)$. Subsequently, the cells were incubated with $5 \mu 1$ Annexin-V-FITC at $5^{\circ} \mathrm{C}$ for $10 \mathrm{~min}$ in dark and then $5 \mu \mathrm{l} \mathrm{PI}$ was added and the cells were incubated at $5^{\circ} \mathrm{C}$ for $10 \mathrm{~min}$ in dark. A flow cytometer (FC500; Beckman Coulter Inc.) was used to detect the apoptosis rate of early apoptotic cells with a analyzing software (EXPO32 ADC v1.2; Beckman Coulter Inc.).

Hippocampus cells from the right hippocampi of the 18 aforementioned rats were collected in the way described above and suspended in $3 \mathrm{ml}$ Dulbecco's modification Eagle's medium (DMEM) and made into a single cell suspension loaded $\left(1-5 \times 10^{5} / 1\right)$ to measure the $\left[\mathrm{Ca}^{2+}\right]_{c}$. Next, $3 \mu$ Fluo-3/AM (calcium ion fluorescence probe; Hangzhou MultiSciences (Lianke) Biotech, Co., Ltd.) was added and cells were incubated at $37^{\circ} \mathrm{C}$ for $30 \mathrm{~min}$, washed twice with DMEM, and then resuspended in DMEM at $37^{\circ} \mathrm{C}$ for $15 \mathrm{~min}$. Flow cytometry instrument (FC500; Beckman Coulter Inc.) was used to measure fluorescence intensity, with the excitation wavelength of $488 \mathrm{~nm}$ and emission wavelength of $525 \mathrm{~nm}$ with a analyzing software (EXPO32 ADC v1.2; Beckman Coulter Inc.).

Western blotting. Western blotting was performed to determine expression of bcl-2, bax and active caspase-3 in the hippocampus. The proteins were extracted from the hippocampal tissue samples of the 6 remainder rats of each group. Briefly, the hippocampus was homogenized in RIPA lysis buffer (Wuhan Servicebio Technology Co., Ltd.), and the total protein concentration of the supernatant was determined using a BCA protein quantification kit (Wuhan Servicebio Technology Co., Ltd.). Proteins were separated by $12 \%$ SDS/PAGE gel (SDS-PAGE kit; Beijing Zoman Biotechnology Co., Ltd.) with $50 \mu$ g protein loaded per lane and then transferred on to PVDF membranes (EMD Millipore). After blocking with $5 \%$ skimmed milk at $37^{\circ} \mathrm{C}$ for $2 \mathrm{~h}$, the membranes were incubated at $4^{\circ} \mathrm{C}$ overnight with primary antibodies: bcl-2 (1:1,000; Proteintech Group Inc.; cat. no. 12789-1-AP), bax (1:1,000; Arigo Biolaboratories Corp.; cat. no. ARG66247), active caspase-3 (1:2,000; ImmunoWay Biotechnology Company; cat. no. YM3431). Anti- $\beta$-actin (1:1,000; ProteinTech Group Inc.; cat. no. 66009-1-lg) was also used as a protein loading control for each sample. Membranes were further incubated with secondary antibodies $(1: 10,000$; Rockland Immunochemicals Inc.; cat. no. 36595) for $2 \mathrm{~h}$ at room temperature. The signals were detected using an Odyssey CLx infrared imaging system (LI-COR Biosciences). ImageJ software (v1.8.0; National Institutes of Health) was used for quantification of the signals.

Statistical analysis. Statistical analyses were performed with the SPSS software v.23.0 (IBM Corp), and the data were expressed as mean $\pm \mathrm{SD}$. Three biological repetitions were conducted for each experiment. Escape latency were analyzed by two-way repeated-measures ANOVA followed by the post hoc Bonferronis test. The statistical differences of all the other data were analyzed by one-way ANOVA without repeated measures followed by the post hoc Bonferronis test. $\mathrm{P}<0.05$ was considered to indicate a statistically significant difference.

\section{Results}

Effects of NBO-PC on cognitive deficit induced by sevoflurane. To evaluate the cognitive function of the rats the MWM test was conducted. All rats had a tendency of reduced escape latency as training progressed indicating that the rats were learning from the day by day practice (Fig. 2A and B). 

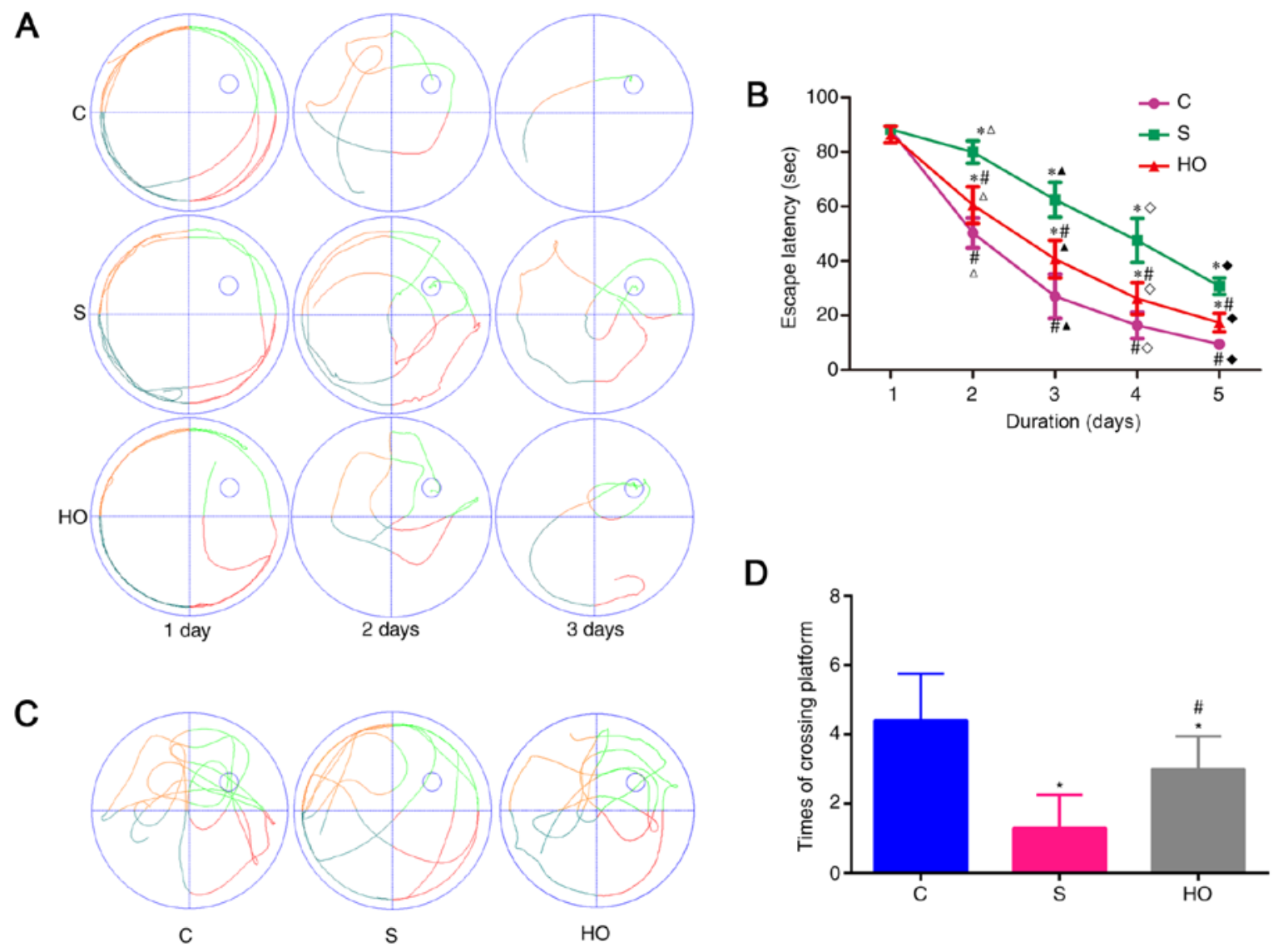

Figure 2. Effects of normobaric hyperoxia preconditioning on sevoflurane-induced learning and memory impairment by the MWM test. (A) Typical paths during place navigation test for each group on days, 1, 3 and 5 after anesthesia. (B) Escape latency (time to find the hidden platform) plotted against training day. 1, 2, 3, 4 and $5 \mathrm{~d}$ means the 1-5 day after rats were anesthetized. Escape latency in each group decreased significantly $(\mathrm{P}<0.05)$ compared with $1 \mathrm{~d}$. (C) A typical path during the probe trial for each group. (D) Platform crossing times during the probe trial of the MWM test. Sevoflurane exposure increased the times of platform crossing compared with the control condition $\left({ }^{*} \mathrm{P}<0.05\right)$. Normobaric hyperoxia preconditioning attenuated the sevoflurane-induced decrease in the number of platform crossings $\left({ }^{*} \mathrm{P}<0.05\right.$ vs. $\mathrm{S}$ group). (n=10/group). ${ }^{*} \mathrm{P}<0.05$ vs. control group, ${ }^{*} \mathrm{P}<0.05$ vs. $\mathrm{S}$ group, ${ }^{\Delta} \mathrm{P}<0.05$ vs. $1 \mathrm{~d},{ }^{\Delta} \mathrm{P}<0.05$ vs. $2 \mathrm{~d}$, ${ }^{\circ} \mathrm{P}<0.05$ vs. $3 \mathrm{~d},{ }^{\circ} \mathrm{P}<0.05$ vs. 4 d. MWM, Morris water maze; $\mathrm{C}$, control group; $\mathrm{S}$ group, sevoflurane group; HO group, sevoflurane + NBO-PC group; NBO-PC, normobaric hyperoxia preconditioning.

The escape latency was significantly longer and the platform crossings were lower in $\mathrm{S}$ group rats compared with $\mathrm{C}$ group rats, indicating significant cognitive impairment induced by sevoflurane (Fig. 2B-D). Notably, the learning and memory impairment induced by sevoflurane was ameliorated by NBO-PC, as indicated by shorter escape latency (Fig. 2B) and increased platform crossings (Fig. $2 \mathrm{C}$ and $\mathrm{D}$ ). Taken together, these results imply that NBO-PC can ameliorate the dysfunction of learning and memory induced by sevoflurane in aged rats.

Effects of NBO-PC on apoptosis in the hippocampus. Next, to determine whether the neuroprotection of NBO pretreatment is associated with the level of apoptosis in the hippocampus, the apoptosis rate in the hippocampus was measured by flow cytometry $24 \mathrm{~h}$ after sevoflurane-exposure. Compared with $\mathrm{C}$ group, the apoptosis rate was significantly increased in the $\mathrm{S}$ and $\mathrm{HO}$ groups (Fig. 3A and B). However, the increase of apoptosis rate in the $\mathrm{HO}$ group was lower compared with that in the $\mathrm{S}$ group (Fig. 3A and B).

In addition, the protein expression level of bcl-2, bax and active caspase- 3 in the hippocampus were also tested to estimate the level of apoptosis. Downregulation of antiapoptosis protein (bcl-2) (30) and upregulation of pro-apoptotic protein (bax) (31) and apoptosis protein (caspases-3) (32) are an indication of apoptosis. Caspase-3 has classically been defined as the main executioner of apoptosis, and the activated form of caspase-3 is involved in the execution phase of apoptosis (33). In the present study, the 'caspase-3' in Fig. 3C and D represented active caspase- 3 .

As shown in Fig. 3C and D, compared with the $\mathrm{C}$ group, the anesthesia with $2.5 \%$ sevoflurane (S group) induced significant increase of bax and active caspase- 3 and a decrease of bcl-2 in the hippocampus. Although similar variations were observed in the $\mathrm{HO}$ group, the change in the $\mathrm{HO}$ group was lower compared with the S group (Fig. 3C and D). All the aforementioned data suggested that NBO-PC inhibited apoptosis in the hippocampus caused by sevoflurane.

Effects of NBO-PC on $\left[\mathrm{Ca}^{2+}\right]_{c}$ in the hippocampus. It has been confirmed that sevoflurane increases $\left[\mathrm{Ca}^{2+}\right]_{c}$ to induce neuroapoptosis (34). To evaluate the role of $\mathrm{Ca}^{2+}$ in the NBO-PC mediated neuroprotective effect, the $\left[\mathrm{Ca}^{2+}\right]_{\mathrm{c}}$ in the hippocampus was measured after sevoflurane anesthesia in the aged rats. Sevoflurane induced an elevation of $\left[\mathrm{Ca}^{2+}\right]_{\mathrm{c}}$ compared with the $\mathrm{C}$ group (Fig. 4A and B), while NBO-PC (HO group) 
A
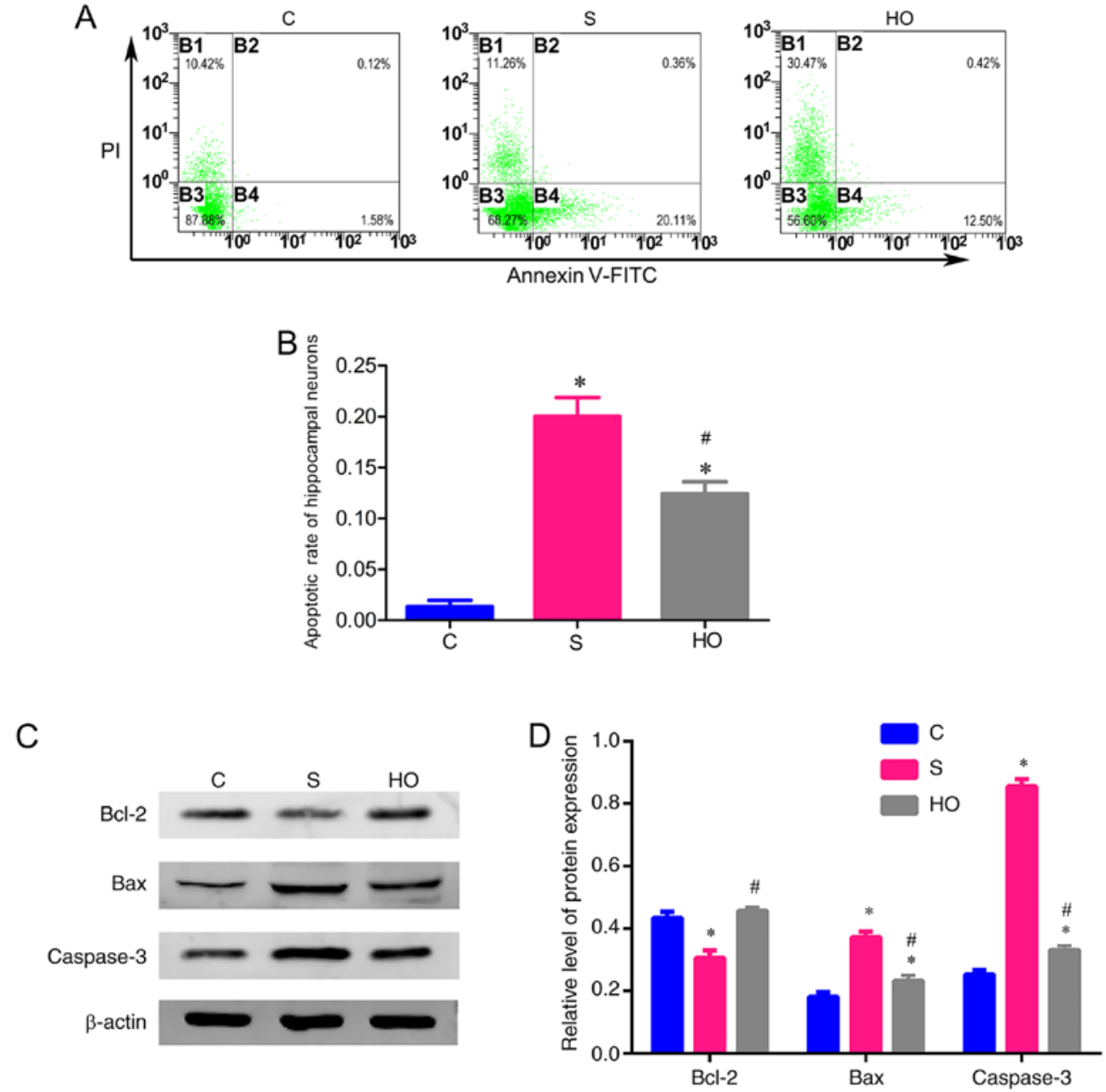

Figure 3. Effects of normobaric hyperoxia preconditioning on the apoptosisin the hippocampus. (A) Representative plots of apoptosis detection by flow cytometry in the hippocampus of aged rats in each group, early apoptotic cells (B4, Annexin- $\left.\mathrm{V}^{+} / \mathrm{PI}\right)$, late apoptotic or necrotic cells $\left(\mathrm{B} 2\right.$, Annexin- $\left.-\mathrm{V}^{+} / \mathrm{PI}^{+}\right)$, live cells (B3, Annexin-V/PI-), mechanical damaged cells (B1, Annexin- $\mathrm{V}^{-} / \mathrm{PI}^{+}$). (B) Apoptosis rate in the hippocampus $24 \mathrm{~h}$ after sevoflurane exposure in each group. (C) Representative western blot image from each group. (D) Western blot analysis of Bcl-2, Bax and active caspase-3 expression in the hippocampus of each group. ${ }^{*} \mathrm{P}<0.05$ vs. control group, ${ }^{\#} \mathrm{P}<0.05$ vs. $\mathrm{S}$ group. $\mathrm{C}$, control group; $\mathrm{S}$ group, sevoflurane group; $\mathrm{HO}$ group, sevoflurane + NBO-PC group; NBO-PC, normobaric hyperoxia preconditioning.
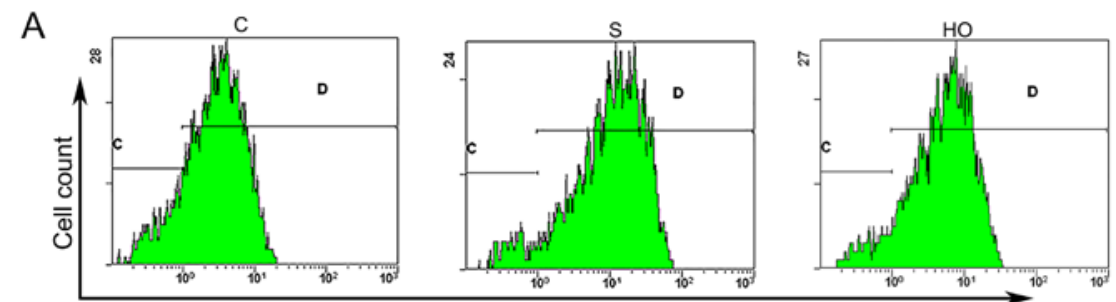

Fluorescence intensity

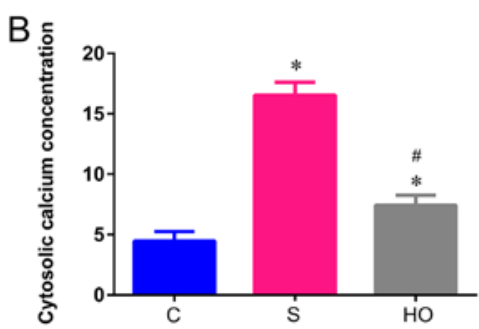

Figure 4. Effects of normobaric hyperoxia preconditioning on the $\left[\mathrm{Ca}^{2+}\right]_{\mathrm{c}}$ in the hippocampus. (A) Intracellular calcium homeostasis in hippocampus cells $24 \mathrm{~h}$ after sevoflurane exposure in each group. (B) Representative histograms of $\left[\mathrm{Ca}^{2+}\right]_{\mathrm{c}}$ by flow cytometry in the hippocampus of aged rats in each group. The ' $C$ ' and ' $D$ ' gates in the figure represent negative and positive cells respectively. ${ }^{*} \mathrm{P}<0.05$ vs. control group, ${ }^{\#} \mathrm{P}<0.05$ vs. $\mathrm{S}$ group. $\left[\mathrm{Ca}^{2+}\right]_{\mathrm{c}}$ cytosolic calcium concentration; C, control group; S group, sevoflurane group; HO group, sevoflurane + NBO-PC group; NBO-PC, normobaric hyperoxia preconditioning; PI, propidium iodide. 
significantly decreased the elevation of $\left[\mathrm{Ca}^{2+}\right]_{c}$ caused by sevoflurane (Fig. 4A and B). Hence, it was proposed that the inhibition of apoptosis induced by NBO-PC was mediated by reducing $\left[\mathrm{Ca}^{2+}\right]_{c}$.

\section{Discussion}

The present study investigated whether NBO-PC ameliorates cognitive deficit after sevoflurane anesthesia, and whether NBO-PC influences the hippocampal apoptosis in aged rats. The major findings of the present study are as follows: i) NBO-PC reduced cognitive deficit induced by sevoflurane-exposure; ii) antiapoptosis protein (bcl-2) expression increased, pro-apoptotic protein (bax) and apoptosis protein (caspase-3) decreased in the hippocampus after NBO-PC; and iii) the apoptosis rate and $\left[\mathrm{Ca}^{2+}\right]_{\mathrm{c}}$ decreased in the hippocampus after NBO-PC. These results demonstrated that the protective role of NBO-PC in sevoflurane-induced spatial and learning impairment was partly related to inhibition of apoptosis in the hippocampus via reducing $\left[\mathrm{Ca}^{2+}\right]_{\mathrm{c}}$.

The potential detrimental effects of anesthetics on aged brains has become a hot topic in recent years due to concerns about the safety of general anesthesia/anesthetics (35). Although clinical evidence regarding the association between anesthetic exposures of aged patients and subsequent cognitive impairments remains unclear, repeated or consistent exposures to general anesthetics may be a potential harmful risk in aged brains $(36,37)$. Sevoflurane with properties of fast onset and rapid recovery (38) is commonly used as a general anesthesia drug in various types of surgery (for example, orthopedic surgery, gynecological surgery and gastrointestinal surgery).

Notably, numerous in vitro and in vivo studies have demonstrated that sevoflurane induces neurotoxicity $(12,15,39)$. Associated with sevoflurane-induced cell injury and death in neurocyte (40), sevoflurane also has been demonstrated to impair the cognitive functions of the aged animals $(12,41)$. Mechanisms underlying sevoflurane-induced neurotoxicity have not been clarified (40), nevertheless, neuroapoptosis may be a key mechanism $(15,42)$. An in vitro study demonstrated that sevoflurane induces apoptosis of neural stem cells by activating $\gamma$-aminobutyric acid (43). Chen et al (44) found that sevoflurane initiates endoplasmic reticulum stress mediated apoptosis in hippocampal neurons of aging rats. The present study revealed that $2.5 \%$ sevoflurane exposure for $5 \mathrm{~h}$ decreased the expression of bcl-2, increased the expression of bax and caspase- 3 , and increased the apoptosis rate in the hippocampus, which were consistent with the changes in cognitive function. Additionally, in the present study the increased $\left[\mathrm{Ca}^{2+}\right]_{\mathrm{c}}$ in the hippocampus after sevoflurane-exposure was also found indicating a calcium-mediated neuroapoptosis. Apart from neuroapoptosis, there are other mechanisms underlying-induced neurotoxicity, such as neuroinflammation (12), neurodegeneration (45).

$\mathrm{NBO}$, in addition to serving as a tool for enhancement of oxygen delivery, has been demonstrated to provided neuroprotection in various models, including ischemia-reperfusion brain injury (18), newborn hypoxia-ischemia brain injury (46), cerebral hemorrhage (47) and brain trauma (48). Notably, Gao et al (21) found that NBO treatment improves spatial learning and memory deficits in APP/PS1 transgenic mice, suggesting that the NBO treatment may have a similar effect on cognitive impairment caused by exposure to anesthetics. Hence, the present study, investigated the effect of NBO pretreatment on the neurotoxicity of sevoflurane in aged rats. In one previous dose-response study (18), protective effect against cerebral ischemia-reperfusion injury was induced by exposing rats to a normobaric hyperoxic environment ( $95 \% \mathrm{O}_{2}$ exposure for 16 and $24 \mathrm{~h}$ consecutively), with no protective effect of NBO exposure less than $16 \mathrm{~h}$. Simultaneously, attention should be paid to the toxicity of long-term oxygen inhalation. It has previously been reported that exposure to $95 \% \mathrm{O}_{2}$ for $24 \mathrm{~h}$ resulted in severe pulmonary congestion with extravasations of red blood cell, edema and alteration in the alveolar structure (49). Mohammadi and Bigdeli (24) confirmed that normobaric hyperoxia preconditioning (exposure to $95 \%$ inspired NBO for $4 \mathrm{~h} / \mathrm{day}$ for 6 consecutive days) had neuroprotective effect on rats in the middle cerebral artery occlusion model. Hence, the regimen for NBO-PC in the present study was based on the current study. The findings of the present study demonstrated that NBO-PC was neuroprotective in aged rats exposed to $2.5 \%$ sevoflurane for $5 \mathrm{~h}$. The MWM test clearly demonstrated that NBO-PC greatly improved cognition impairment caused by sevoflurane in aged rats. As expected, the apoptosis in the hippocampus was in concert with the changes in cognitive function in the present study. In addition, the present study demonstrated that antiapoptosis protein (bcl-2) expression increased, pro-apoptotic protein (bax) and apoptosis protein (caspases-3) decreased in the hippocampus after NBO-PC, and the apoptosis rate decreased in the hippocampus after NBO-PC.

Concurrently, it was observed in the present study that NBO-PC reduced the increase of $\left[\mathrm{Ca}^{2+}\right]_{\mathrm{c}}$ to sevoflurane in the hippocampus. Intracellular $\mathrm{Ca}^{2+}$, one of the most widely used intracellular messengers is involved in controlling almost all cell processes including muscle contraction, exocytosis, proliferation, differentiation, protein synthesis and gene expression (50). Disruption of the intracellular calcium homeostasis, particularly due to a persistent and excessive increase in the intracellular $\mathrm{Ca}^{2+}$ can induce cell death by apoptosis (51). In addition, apoptosis cell death mediated by calcium dysregulation serves an important role in anesthetic neurotoxicity (52). The results of the present study implied that NBO-PC may play a neuroprotective role by reducing the intracellular calcium increase caused by sevoflurane anesthesia through some molecular mechanisms, which need further study.

In the present study, a lower chloral hydrate dose $(250 \mathrm{mg} / \mathrm{kg}$ ) was used to anaesthetize the rats in accordance with some previous studies $(53,54)$. Some studies $(55,56)$ use $300-400 \mathrm{mg} / \mathrm{kg}$ chloral hydrate to perform surgery or establish animal models, the process of which requires deep anesthesia and long duration. However, in the present study just a mild anesthesia was needed to ensure that the rats were hypnotized without struggling. In addition, the potential effects of chloral hydrate on experimental variables should also be taken into consideration (57). Therefore, in view of the low requirements for the depth and the duration of anesthesia and the possible influence of high dose of chloral hydrate on detection indicators, the lower dose of chloral hydrate was used to anaesthetize the rats in the present study. 
The present study had some limitations. In the present study, 20-month-old rats were selected as aged rats. Among the published articles on cognitive dysfunction, the ages of old rats were very different ranging from 18-24 months (58-65). According to the aforementioned studies, the rats selected by the present study are equivalent to the aged. It was reported that 1 rat month is comparable to 3 human years in adulthood (66), which indicates differences in anatomy, physiology and developmental processes of different months old rats. In addition, these differences may lead to differences in research results. Although the precise correlation between age of laboratory rats and human is still a subject of debate, it has been accepted that 24 month old rats correspond to 60 year old humans (66). Hence, the age of the old rats in this experiment may be relatively young. In addition, some assays, such as histopathological staining of the hippocampal sections were not performed to visually reflect the brain damage caused by sevoflurane due to funding. So future studies must select much older rats as subjects and optimize the assessment of brain damage through histopathological staining if funding is available.

In conclusion, the present study demonstrated that NBO-PC decreased the hippocampal apoptosis as well as alleviated the memory deficits in aged rats who were exposed to sevoflurane. The results of the present study, suggested that neuron apoptosis inhibited by NBO-PC is in association with a decrease of cytosolic $\mathrm{Ca}^{2+}$ in the hippocampus, which may at least partially be the molecular mechanism by which NBO-PC induces neuroprotection.

\section{Acknowledgements}

Not applicable.

\section{Funding}

The present study was supported by grants from the National Natural Science Foundation of China (grant no. 81771134), the Natural Science Foundation of Hebei Province (grant no. H2018206305), the Hebei Province Technology Innovation guide Project Science and the Technology Winter Olympics Special Project (grant no. 19977790D). The Hebei Provincial government funded the specialty capacity building and specialty leader training program.

\section{Availability of data and materials}

The datasets used and/or analyzed during the current study are available from the corresponding author on reasonable request.

\section{Authors' contributions}

YW was responsible for designing the study, performing the experiment, collecting the data and writing the manuscript. CPY was responsible for designing the study, performing the experiment, and collecting the data. YLT and ZJZ were responsible for collection of experimental specimens and the extraction of proteins and reviewing the manuscript. $\mathrm{ZYH}$ was responsible for analyzing and interpreting the data.
QJW was responsible for providing experimental ideas and reviewing the manuscript. YW and QJW were responsible for the confirming the authenticity of all the raw data. All authors read and approved the final manuscript.

\section{Ethics approval and consent to participate}

This study was approved by the Ethics Committee for Animal Experimentation (Ethical approval no. Guo A2017-026-1), and the animals were studied at Hebei Medical University (Shijiazhuang, China). All applicable international, national, and/or institutional guidelines for the care and use of animals were followed.

\section{Patient consent for publication}

Not applicable.

\section{Competing interests}

The authors declare that they have no competing interests.

\section{References}

1. Xu X, Hu Y, Yan E, Zhan G, Liu C and Yang C: Perioperative neurocognitive dysfunction: Thinking from the gut? Aging 12: 15797-15817, 2020.

2. Kotekar N, Shenkar A and Nagaraj R: Postoperative cognitive dysfunction-current preventive strategies. Clin Interv Aging 13: 2267-2273, 2018.

3. Moller JT, Cluitmans P, Rasmussen LS, Houx P, Rasmussen H, Canet J, Rabbitt P, Jolles J, Larsen K, Hanning CD, et al: Long-term postoperative cognitive dysfunction in the elderly ISPOCD1 study. ISPOCD investigators. International study of post-operative cognitive dysfunction. Lancet 351: 857-861, 1998.

4. Liu Y and Yin Y: Emerging roles of immune cells in postoperative cognitive dysfunction. Mediators Inflamm 2018: 6215350 2018.

5. Berger M, Nadler JW, Browndyke J, Terrando N, Ponnusamy V, Cohen HJ, Whitson HE and Mathew JP: Postoperative cognitive dysfunction: Minding the gaps in our knowledge of a common postoperative complication in the elderly. Anesthesiol Clin 33: 517-550, 2015.

6. Vide S and Gambús PL: Tools to screen and measure cognitive impairment after surgery and anesthesia. Presse Med 47: e65-e72, 2018.

7. Ologunde R and Ma D: Do inhalational anesthetics cause cognitive dysfunction? Acta Anaesthesiol Taiwan 49: 149-153, 2011.

8. Zhang F, Zhu ZQ, Liu DX, Zhang C, Gong QH and Zhu YH: Emulsified isoflurane anesthesia decreases brain-derived neurotrophic factor expression and induces cognitive dysfunction in adult rats. Exp Ther Med 8: 471-477, 2014.

9. Chen Y, Zhang P, Lin X, Zhang H, Miao J, Zhou Y and Chen G: Mitophagy impairment is involved in sevoflurane-induced cognitive dysfunction in aged rats. Aging (Albany NY) 12: 17235-17256, 2020 (Online ahead of print).

10. Tojo A, Uchimoto K, Inagawa G and Goto T: Desflurane impairs hippocampal learning on day 1 of exposure: A prospective laboratory study in rats. BMC Anesthesiol 19: 119, 2019.

11. Zhang S, Hu X, Guan W, Luan L, Li B, Tang Q and Fan H: Isoflurane anesthesia promotes cognitive impairment by inducing expression of $\beta$-amyloid protein-related factors in the hippocampus of aged rats. PLoS One 12: e0175654, 2017.

12. Cui RS, Wang K and Wang ZL: Sevoflurane anesthesia alters cognitive function by activating inflammation and cell death in rats. Exp Ther Med 15: 4127-4130, 2018.

13. Istaphanous GK, Howard J, Nan X, Hughes EA, McCann JC, McAuliffe JJ, Danzer SC and Loepke AW: Comparison of the neuroapoptotic properties of equipotent anesthetic concentrations of desflurane, isoflurane, or sevoflurane in neonatal mice. Anesthesiology 114: 578-587, 2011. 
14. Steinmetz J, Christensen KB, Lund T, Lohse N and Rasmussen LS; ISPOCD Group: Long-term consequences of postoperative cognitive dysfunction. Anesthesiology 110: 548-555, 2009.

15. Liu X, Song X, Yuan T, He J, Wang X and Wang Q: Effects of calpain on sevoflurane-induced aged rats hippocampal neuronal apoptosis. Aging Clin Exp Res 28: 633-639, 2016.

16. Cao L, Li L, Lin D and Zuo Z: Isoflurane induces learning impairment that is mediated by interleukin $1 \beta$ in rodents. PLoS One 7: e51431, 2012.

17. Liang G, Ward C, Peng J, Zhao Y, Huang B and Wei $\mathrm{H}$ : Isoflurane causes greater neurodegeneration than an equivalent exposure of sevoflurane in the developing brain of neonatal mice. Anesthesiology 112: 1325-1334, 2010.

18. Bigdeli MR, Rasoulian B and Meratan AA: In vivo normobaric hyperoxia preconditioning induces different degrees of antioxidant enzymes activities in rat brain tissue. Eur J Pharmacol 611 22-29, 2009.

19. Petrosillo G, Di Venosa N, Moro N, Colantuono G, Paradies V, Tiravanti E, Federici A, Ruggiero FM and Paradies G: In vivo hyperoxic preconditioning protects against rat-heart ischemia/reperfusion injury by inhibiting mitochondrial permeability transition pore opening and cytochrome c release. Free Radic Biol Med 50: 477-483, 2011.

20. Wahhabaghai H, Heidari R, Zeinoddini A, Soleyman-Jahi S Golmanesh L, Rasoulian B, Akbari H, Foadoddoni M and Esmailidehaj M: Hyperoxia-induced preconditioning against renal ischemic injury is mediated by reactive oxygen species but not related to heat shock proteins 70 and 32. Surgery 157: 1014-1022, 2015.

21. Gao B, Long Z, Zhao L and He G: Effect of normobaric hyperoxia on behavioral deficits and neuropathology in Alzheimer's disease mouse model. J Alzheimers Dis 27: 317-326, 2011.

22. Bigdeli MR: Neuroprotection caused by hyperoxia preconditioning in animal stroke models. ScientificWorldJournal 11 403-421, 2011.

23. Ma H, Yao L, Pang L, Li X and Yao Q: Tetrandrine ameliorates sevoflurane-induced cognitive impairment via the suppression of inflammation and apoptosis in aged rats. Mol Med Rep 13: 4814-4820, 2016.

24. Mohammadi E and Bigdeli MR: Effects of preconditioning with normobaric hyperoxia on $\mathrm{Na}^{+} / \mathrm{Ca}^{2+}$ exchanger in the rat brain Neuroscience 237: 277-284, 2013.

25. Banfalvi G: Methods to detect apoptotic cell death. Apoptosis 22: 306-323, 2017

26. Riccardi C and Nicoletti I: Analysis of apoptosis by propidium iodide staining and flow cytometry. Nat Protoc 1: 1458-1461, 2006.

27. Wlodkowic D, Skommer J and Darzynkiewicz Z: Flow cytometry-based apoptosis detection. Methods Mol Biol 559: 19-32. 2009

28. Field KJ, White WJ and Lang CM: Anaesthetic effects of chloral hydrate, pentobarbitone and urethane in adult male rats. Lab Anim 27: 258-269, 1993.

29. Reimer JN, Schuster CJ, Knight CG, Pang DSJ and Leung VSY: Intraperitoneal injection of sodium pentobarbital has the potential to elicit pain in adult rats (Rattus norvegicus). PLoS One 15: e0238123, 2020.

30. Senichkin VV, Pervushin NV, Zuev AP, Zhivotovsky B and Kopeina GS: Targeting Bcl-2 family proteins: What, where, when? Biochemistry (Mosc) 85: 1210-1226, 2020.

31. D'Orsi B, Mateyka J and Prehn JHM: Control of mitochondrial physiology and cell death by the Bcl-2 family proteins Bax and Bok. Neurochem Int 109: 162-170, 2017.

32. Snigdha S, Smith ED, Prieto GA and Cotman CW: Caspase-3 activation as a bifurcation point between plasticity and cell death. Neurosci Bull 28: 14-24, 2012.

33. Chang L, Zhang X, Liu W, Song Y, Gao X, Ling W and $\mathrm{Wu}$ Y: Immunoreactivity of Ki-67/ $\beta$-tubulin and immunocolocalization with active caspase- 3 in rat dentate gyrus during postnatal development. J Chem Neuroanat 46: 10-18, 2012.

34. Zhu X, Yao Y, Guo M, Li J, Yang P, Xu H and Lin D: Sevoflurane increases intracellular calcium to induce mitochondrial injury and neuroapoptosis. Toxicol Lett 336: 11-20, 2021.

35. Vlisides P and Xie Z: Neurotoxicity of general anesthetics: An update. Curr Pharm Des 18: 6232-6240, 2012.

36. Armstrong R, Xu F, Arora A, Rasic N and Syed NI: General anesthetics and cytotoxicity: Possible implications for brain health. Drug Chem Toxicol 40: 241-249, 2017.
37. Manatpon $\mathrm{P}$ and Kofke WA: Toxicity of inhaled agents after prolonged administration. J Clin Monit Comput 32: 651-666, 2018.

38. Ebert TJ, Robinson BJ, Uhrich TD, Mackenthun A and Pichotta PJ: Recovery from sevoflurane anesthesia: A comparison to isoflurane and propofol anesthesia. Anesthesiology 89: 1524-1531, 1998.

39. Lv G, Li C, Wang W, Li N and Wang K: Silencing SP1 alleviated sevofurane-induced pocd development via cholinergic anti-infammatory pathway. Neurochem Res 45: 2082-2090, 2020.

40. Yang L, Shen Q, Xia Y, Lei X and Peng J: Sevoflurane-induced neurotoxicity is driven by OXR1 post-transcriptional downregulation involving has-miR-302e. Mol Med Rep 18: 4657-4665, 2018.

41. Fei X, Wang JX, Wu Y, Dong N and Sheng ZY: Sevofluraneinduced cognitive decline in aged mice: Involvement of toll-like receptors 4. Brain Res Bull 165: 23-29, 2020.

42. Yang X, Zheng YT and Rong W: Sevoflurane induces apoptosis and inhibits the growth and motility of colon cancer in vitro and in vivo via inactivating Ras/Raf/MEK/ERK signaling. Life Sci 239: 116916, 2019

43. Qiu J, Shi P, Mao W, Zhao Y, Liu W and Wang Y: Effect of apoptosis in neural stem cells treated with sevoflurane. BMC Anesthesiol 15: 25, 2015.

44. Chen G, Gong M, Yan M and Zhang X: Sevoflurane induces endoplasmic reticulum stress mediated apoptosis in hippocampal neurons of aging rats. PLoS One 8: e57870, 2013.

45. Jiang $\mathrm{J}$ and Jiang H: Effect of the inhaled anesthetics isoflurane, sevoflurane and desflurane on the neuropathogenesis of Alzheimer's disease (Review). Mol Med Rep 12: 3-12, 2015.

46. Kelestemur T, Beker MC, Caglayan AB, Caglayan B, Altunay S, Kutlu $S$ and Kilic E: Normobaric oxygen treatment improves neuronal survival functional recovery and axonal plasticity after newborn hypoxia-ischemia. Behav Brain Res 379: 112338 , 2020.

47. You P, Lin M, Li K, Ye X and Zheng J: Normobaric oxygen therapy inhibits HIF-1 $\alpha$ and VEGF expression in perihematoma and reduces neurological function defects. Neuroreport 27: 329-336, 2016.

48. Donkin JJ and Vink R: Mechanisms of cerebral edema in traumatic brain injury: Therapeutic developments. Curr Opin Neurol 23: 293-299, 2010

49. Al-Motabagani MA: Histological changes in the alveolar structure of the rat lung after exposure to hyperoxia. Ital J Anat Embryol 110: 209-223, 2005.

50. Berridge MJ, Bootman MD and Roderick HL: Calcium signalling: Dynamics, homeostasis and remodelling. Nat Rev Mol Cell Biol 4: 517-529, 2003.

51. Orrenius S, Zhivotovsky B and Nicotera P: Regulation of cell death: The calcium-apoptosis link. Nat Rev Mol Cell Biol 4: 552-565, 2003.

52. Yang $\mathrm{M}$ and Wei $\mathrm{H}$ : Anesthetic neurotoxicity: Apoptosis and autophagic cell death mediated by calcium dysregulation. Neurotoxicol Teratol 60: 59-62, 2017.

53. Silverman J and Muir WW III: A review of laboratory animal anesthesia with chloral hydrate and chloralose. Lab Anim Sci 43: 210-216, 1993.

54. Uhlig C, Krause H, Koch T, Gama de Abreu M and Spieth P: Anesthesia and monitoring in small laboratory mammals used in anesthesiology, respiratory and critical care research: A systematic review on the current reporting in top-10 impact factor ranked journals. PLoS One 10: e0134205, 2015.

55. Sjakste N, Baumane L, Meirena D, Lauberte L, Dzintare M and Kalvins I: Drastic increase in nitric oxide content in rat brain under halothane anesthesia revealed by EPR method. Biochem Pharmacol 58: 1955-1959, 1999.

56. Grinchii D, Paliokha R, Tseilikman V and Dremencov E: Inhibition of cytochrome $\mathrm{P} 450$ by proadifen diminishes the excitability of brain serotonin neurons in rats. Gen Physiol Biophys 37: 711-713, 2018.

57. Herbst LS, Gaigher T, Siqueira AA, Joca SRL Sampaio KN and Beijamini V: New evidence for refinement of anesthetic choice in procedures preceding the forced swimming test and the elevated plus-maze. Behav Brain Res 368: 111897, 2019.

58. Templer VL, Wise TB and Heimer-McGinn VR: Social housing protects against age-related working memory decline independently of physical enrichment in rats. Neurobiol Aging 75: 117-125, 2019. 
59. Sun J, Zhang S, Zhang X, Dong $\mathrm{H}$ and Qian Y: IL-17A is implicated in lipopolysaccharide-induced neuroinflammation and cognitive impairment in aged rats via microglial activation. J Neuroinflammation 12: 165, 2015.

60. La Spina M, Sansevero G, Biasutto L, Zoratti M, Peruzzo R, Berardi N, Sale A and Azzolini M: Pterostilbene improves cognitive performance in aged rats: An in vivo study. Cell Physiol Biochem 52: 232-239, 2019.

61. Nishigaki A, Kawano T, Iwata H, Aoyama B, Yamanaka D, Tateiwa H, Shigematsu-Locatelli M, Eguchi S, Locatelli FM and Yokoyama M: Acute and long-term effects of haloperidol on surgery-induced neuroinflammation and cognitive deficits in aged rats. J Anesth 33: 416-425, 2019

62. Yang N, Li Z, Han D, Mi XN, Tian M, Liu T, Li Y, He J, Kuang C, Cao Y, et al: Autophagy prevents hippocampal $\alpha$-synuclein oligomerization and early cognitive dysfunction after anesthesia/surgery in aged rats. Aging (Albany NY) 12: 7262-7281, 2020.
63. Cao Y, Li Z, Ma L, Yang N and Guo XY: Isoflurane-induced postoperative neurovascular and cognitive dysfunction is associated with VEGF overexpression in aged rats. J Mol Neurosci 69: 215-223, 2019

64. Terrando N, Yang T, Wang X, Fang J, Cao M, Andersson U, Erlandsson HH, Ouyang W and Tong J: Systemic HMGB1 neutralization prevents postoperative neurocognitive dysfunction in aged rats. Front Immunol 7: 441, 2016.

65. Fonken LK, Frank MG, D'Angelo HM, Heinze JD, Watkins LR, Lowry CA and Maier SF: Mycobacterium vaccae immunization protects aged rats from surgery-elicited neuroinflammation and cognitive dysfunction. Neurobiol Aging 71: 105-114, 2018.

66. Sengupta P: The laboratory rat: Relating its age with human's. Int J Prev Med 4: 624-630, 2013.

This work is licensed under a Creative Commons Attribution-NonCommercial-NoDerivatives 4.0 International (CC BY-NC-ND 4.0) License. 